\title{
Thin Film Polymer Composite Scintillators for Thermal Neutron Detection
}

\author{
Andrew N. Mabe, ${ }^{1}$ John D. Auxier II, ${ }^{1}$ Matthew J. Urffer, ${ }^{2}$ Stephen A. Young, \\ Dayakar Penumadu, ${ }^{4}$ George K. Schweitzer, ${ }^{1}$ and Laurence F. Miller ${ }^{2}$
}

${ }^{1}$ Department of Chemistry, University of Tennessee, Knoxville, TN 37996, USA

${ }^{2}$ Department of Nuclear Engineering, University of Tennessee, Knoxville, TN 37996, USA

${ }^{3}$ Engineering Science and Mechanics, University of Tennessee, Knoxville, TN 37996, USA

${ }^{4}$ Civil and Environmental Engineering, University of Tennessee, Knoxville, TN 37996, USA

Correspondence should be addressed to Andrew N. Mabe; andrew.n.mabe@gmail.com

Received 4 February 2013; Accepted 15 April 2013

Academic Editor: Hui Shen Shen

Copyright (C) 2013 Andrew N. Mabe et al. This is an open access article distributed under the Creative Commons Attribution License, which permits unrestricted use, distribution, and reproduction in any medium, provided the original work is properly cited.

\begin{abstract}
Thin film polystyrene composite scintillators containing ${ }^{6} \mathrm{LiF}$ and organic fluors have been fabricated and tested as thermal neutron detectors. Varying fluorescence emission intensities for different compositions are interpreted in terms of the Beer-Lambert law and indicate that the sensitivity of fluorescent sensors can be improved by incorporating transparent particles with refractive index different than that of the polymer matrix. Compositions and thicknesses were varied to optimize the fluorescence and thermal neutron response and to reduce gamma-ray sensitivity. Neutron detection efficiency and neutron/gamma-ray discrimination are reported herein as functions of composition and thickness. Gamma-ray sensitivity is affected largely by changing thickness and unaffected by the amount of ${ }^{6} \mathrm{LiF}$ in the film. The best neutron/gamma-ray discrimination characteristics are obtained for film thicknesses in the range $25-150 \mu \mathrm{m}$.
\end{abstract}

\section{Introduction}

The development of efficient thermal neutron detectors is relevant to the fields of nuclear physics, nuclear power generation, imaging, and homeland security. Helium-3 filled proportional counters are widely used in radiation portal monitors to detect illicit transport of fissile materials, in neutron scattering experiments, in medical imaging, and in well logging. Due to the recent expanded use and reduced production of He-3, the supply of He-3 is dwindling such that the Department of Homeland Security has issued research funds to develop a replacement technology [1]. As described herein, efforts have been made to develop inexpensive and atmospherically stable polymeric composite materials to function as thermal neutron scintillation detectors that have low sensitivity to gamma-rays.

The use of organic polymers as scintillators has many advantages over other scintillating materials such as single crystals and inorganic glasses in that selected polymers are air-stable, do not require high processing temperatures, have relatively low cost, are easy to fabricate in large areas in a wide range of geometries, and have fast response times [2]. Common commercially available polymeric scintillators are based on aryl vinyl polymers such as polystyrene (PS) and polyvinyltoluene (PVT). The aromatic pendant groups on these polymers have emission in the wavelength range 300-350 $\mathrm{nm}$ under both UV- and X-ray induced excitation $[3,4]$, so these polymers must be doped with wavelength shifters to shift the wavelength of scintillation to the region of sensitivity of common bialkali photomultiplier tubes (PMTs) (390-450 nm).

In order for organic polymers to function as thermal neutron detectors, they must be sensitized with neutron capture nuclides such as ${ }^{6} \mathrm{Li},{ }^{10} \mathrm{~B}$, or ${ }^{157} \mathrm{Gd}$ [5]. In this study, ${ }^{6} \mathrm{Li}$ was selected as the thermal neutron capture nuclide because of its large capture cross section ( 940 barns) and large reaction energy $(Q=4.78 \mathrm{MeV})$. On absorption of a thermal 
neutron, ${ }^{6} \mathrm{Li}$ fissions into an alpha particle $(2.05 \mathrm{MeV})$ and a triton $(2.73 \mathrm{MeV})$ [6]. These charged particles deposit their kinetic energy in the matrix primarily by electromagnetic interactions with bound electrons to form ionizations and excitations in the scintillator matrix. The excitations are then collected by fluors which shift the emission wavelength to a region suitable to be collected by a PMT.

In our previous work, we evaluated poly(2-vinyl naphthalene) containing ${ }^{6}$ lithium salicylate $\left({ }^{6} \mathrm{LiSal}\right)$ and conjugated organic dyes [7] and poly(ethylene naphthalate) containing ${ }^{6}$ lithium fluoride $\left({ }^{6} \mathrm{LiF}\right)$ and conjugated organic dyes [8] as potential thermal neutron detectors. In this study, ternary composite films comprising PS, ${ }^{6} \mathrm{LiF}$, and glass-forming organic dyes were explored as potential thermal neutron scintillation detectors. Several metrics were used to evaluate the performance of the detectors. Intrinsic neutron detection efficiency $\left(\varepsilon_{\text {int, } n}\right)$ is defined as fraction of neutrons passing through the surface of the detector that are detected (1). Intrinsic gamma-ray detection efficiency $\left(\varepsilon_{\text {int, },}\right)$ is defined as fraction of gamma-rays passing through the surface of the detector that are detected (2). Gamma-neutron detection efficiency is defined herein as the number of gamma-rays that are misclassified as neutrons. These criteria are used because they are independent of the area of the detector. Common detector systems based on He-3 have gammaneutron detection efficiencies of $1.7 \times 10^{-9}$ and absolute neutron detection efficiencies of 2.83 counts per second per nanogram of ${ }^{252} \mathrm{Cf}[9]$. Herein results are discussed in terms of intrinsic efficiencies because this quantity is independent of the size of the detector

$$
\varepsilon_{\text {int,n }}=
$$

Number of neutrons detected

Number of neutrons passing across detector surface,

$$
\varepsilon_{\text {int }, \gamma}=
$$

Number of gamma-rays detected

Number of gamma-rays passing across detector surface

\section{Materials and Methods}

2.1. Component Selection. An ideal thermal neutron scintillation detector is stable in atmospheric conditions such that it is not significantly damaged through the course of common use. Incorporation of hygroscopic materials into a hydrophobic polymeric matrix causes water to be absorbed in the material which decreases the mechanical integrity of the composite over time. Many lithium salts are hygroscopic which sharply narrows the available lithiated materials that can be implemented as neutron sensitizers. In the present work, lithium fluoride was chosen because it is not hygroscopic, does not decompose in the presence of the atmosphere, is thermally stable, and is transparent in the wavelength region $300-450 \mathrm{~nm}$. Moreover, it has an atom density of $6.117 \times 10^{22}$ lithium atoms $/ \mathrm{cm}^{3}$ (corrected for $97.6 \%{ }^{6} \mathrm{Li}$ enrichment) which is higher than most common lithium salts. Utilization of a higher atom density material decreases the volume fraction of neutron activator material required to achieve the desired neutron detection efficiency. PS was selected because it is commonly implemented as a matrix for polymer scintillators, it is comparably inexpensive, and it is easy to synthesize. ${ }^{6}$ Lithium fluoride was synthesized in our laboratory rather than purchasing because the cost of synthesizing it in our laboratory is lower than that required to purchase enriched lithium fluoride.

2.2. Materials. Lithium-6 hydroxide monohydrate enriched to $97.6 \%{ }^{6} \mathrm{Li}$ was heated to $110^{\circ} \mathrm{C}$ for four hours to remove volatile impurities, dissolved in deionized water, and filtered with a $2.5 \mu \mathrm{m}$ filter to remove insoluble impurities. The ${ }^{6} \mathrm{Li} /{ }^{7} \mathrm{Li}$ ratio was determined by QSTAR Mass Spectroscopy. Hydrofluoric acid (48\%, Aldrich) and methanol (Fisher Scientific) were used without further purification. Tetrahydrofuran (Fisher Scientific) was fractionally distilled from calcium chloride immediately before use to remove water and butylated hydroxytoluene stabilizer. Styrene (Acros) was purified immediately before use by passing the monomer through a column containing activated basic alumina on top of silica gel to remove monomethyl ether of hydroquinone (MEHQ) inhibitor and other impurities. Free radical initiator $2,2^{\prime}$-azobisisobutyronitrile (AIBN), obtained from Aldrich, was recrystallized from methanol immediately before use. A preblended fluor mixture comprising 2,5-diphenyloxazole (PPO) and 1,4-bis(5-phenyl-2-oxazolyl)benzene (POPOP) mixed as $97.1 \%$ PPO and 2.87\% POPOP, obtained from Curtiss Laboratories, Inc., was used without further purification. This blend will be referred to as PPO/POPOP. Acrylic disks $3 \mathrm{~mm}$ thick with a diameter of $50.8 \mathrm{~mm}$ (Eljen Technologies) were cleaned with acetone immediately prior to use.

2.3. Synthesis. Lithium-6 fluoride was synthesized in a Teflon beaker by dissolving purified lithium hydroxide in deionized water and adding hydrofluoric acid until the solution was slightly acidic. The solution was stirred for 10 minutes to ensure no lithium hydroxide remained. The resulting precipitate was collected by vacuum filtration using a $2.5 \mu \mathrm{m}$ filter, washed with deionized water to remove soluble impurities, and then dried by washing with acetone. The resulting white powder was stored in a vacuum dessicator until needed.

Polystyrene was synthesized by conventional free radical polymerization. Purified styrene $(10 \mathrm{~mL})$ and AIBN $(60 \mathrm{mg})$ were placed in a vial containing a septum. The container was sealed, degassed three times with vacuum and argon, and placed in an oil bath at $90^{\circ} \mathrm{C}$ to polymerize. After 60 hours, the resulting transparent polymer disk was dissolved in $150 \mathrm{~mL}$ purified tetrahydrofuran, precipitated by dropwise addition into $900 \mathrm{~mL}$ cold methanol, and then collected by vacuum filtration and washed with methanol. The purified polymer was dried in air for at least 24 hours prior to use.

2.4. Film Casting. The compositions of interest, expressed in weight percentages, were $0 \%-30 \%{ }^{6} \mathrm{LiF}, 5 \%-15 \% \mathrm{PPO} /$ POPOP, and thicknesses over the range $15 \mu \mathrm{m}-2.6 \mathrm{~mm}$. To fabricate film samples, measured amounts of PS, ${ }^{6} \mathrm{LiF}$, and 


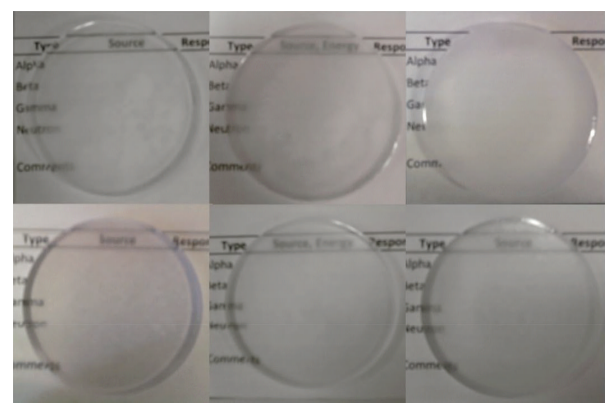

FIGURE 1: Representative images of PS composites containing ${ }^{6} \mathrm{LiF}$ and $\mathrm{PPO} / \mathrm{POPOP}$. Top row: samples with varying thicknesses containing $20 \%{ }^{6} \mathrm{LiF}$ and $5 \% \mathrm{PPO} / \mathrm{POPOP}$. Left to right: $25 \mu \mathrm{m}$, $50 \mu \mathrm{m}$, and $150 \mu \mathrm{m}$. Bottom Row: $15 \mu \mathrm{m}$ samples containing $5 \%$ $\mathrm{PPO} / \mathrm{POPOP}$ and varying concentrations of ${ }^{6} \mathrm{LiF}$. Left to right: $10 \%$, $20 \%$, and $30 \%$.

$\mathrm{PPO} / \mathrm{POPOP}$ were placed in a small glass vial. An appropriate volume of tetrahydrofuran was added to make a $5 \%$ solution which was heated to $60^{\circ} \mathrm{C}$ for 20 minutes to dissolve the polymer and fluors, then sonicated to suspend the ${ }^{6} \mathrm{LiF}$. Each sample was then cast onto an acrylic disk, covered with a beaker to slow evaporation rate and prevent dust contamination and crazing due to drafts, then dried for 24 hours to give films of varying translucency as shown in Figure 1. Samples were characterized without removal from the acrylic disk to prevent stress crazing and mechanical failure of the film, to facilitate coupling to the PMT, and to prevent direct contact of the scintillator with optical grease.

Images demonstrating the relative optical clarity of some of the samples are shown in Figure 1. At a constant concentration of ${ }^{6} \mathrm{LiF}$, thicker samples are less transparent than thinner samples. Increasing the concentration of ${ }^{6} \mathrm{LiF}$ decreases the optical clarity as would be expected due to the difference in refractive indices between the ${ }^{6} \mathrm{LiF}$ and the PS. This decrease in transmission is more highly dependent on the concentration of ${ }^{6} \mathrm{LiF}$ in thicker samples than in thinner samples. The $15 \mu \mathrm{m}$ thick samples do not show much change in transmission over the concentration range of ${ }^{6} \mathrm{LiF}$ studied. The morphology and size distribution of the ${ }^{6} \mathrm{LiF}$ particles in the composites were examined by a Leica SP2 confocal laser-scanning microscope (CLSM) equipped with a $488 \mathrm{~nm}$ argon ion laser to probe the distribution of the particles in the interior volume of the sample. Image processing and measurements were performed with ImageJ software. Cubic ${ }^{6} \mathrm{LiF}$ particles with a mean size of $3.2 \mu \mathrm{m}$ are clearly visible in the image shown in Figure 2. Several images in different places of the sample were obtained. The images demonstrate that the particles are randomly distributed in the matrix and not agglomerated.

2.5. Characterizations. Fluorescence measurements were conducted using a HORIBA Jobin Yvon Fluorolog-3 spectrofluorometer equipped with a $450 \mathrm{~W}$ Xe lamp as the excitation source. Both emission and excitation spectra were collected with $1 \mathrm{~nm}$ spectral bandwidth.

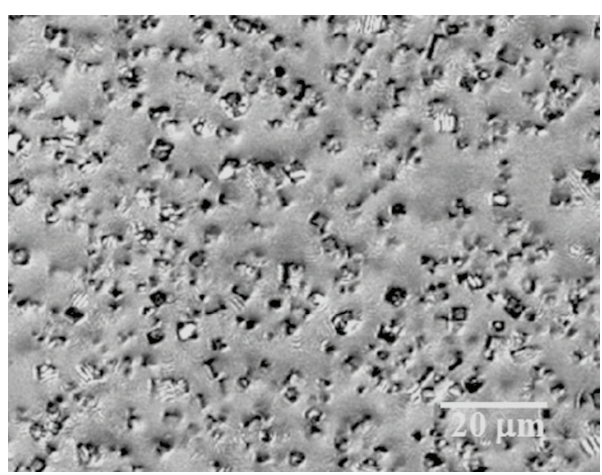

Figure 2: Bright-field CLSM image of ${ }^{6} \mathrm{LiF}$ particles distributed in a PS matrix. Mean particle size $=3.2 \mu \mathrm{m}$, range $=1.6-5.3 \mu \mathrm{m}$. The scale bar is $20 \mu \mathrm{m}$.

Scintillation measurements were conducted as previously described [8]. A diagram of the neutron irradiator is shown in Figure 3. Briefly, samples cast on acrylic disks were coupled to a photomultiplier tube using optical grease with the sample side facing away from the PMT, then covered with a Teflon tape reflector. A moderated mass of $0.59 \mu \mathrm{g}{ }^{252} \mathrm{Cf}$ was used as the neutron source. Thermal neutron response was determined by first irradiating the sample inside an acrylic tube $1.6 \mathrm{~mm}$ thick surrounded by $1.6 \mathrm{~mm}$ of lead to obtain the scintillation response to gamma-rays and all neutrons. The sample was then irradiated in an acrylic tube surrounded by a $1.6 \mathrm{~mm}$ thick sheet of cadmium to shield thermal neutrons and measure the response to gamma-rays and fast neutrons. The number of gamma-rays shielded by the lead tube is similar to the number of gamma-rays shielded by the cadmium tube. Spectral subtraction was then used to obtain the net thermal neutron response. It should be noted that the number of gamma-rays inside the cadmium tube is slightly higher than inside the lead tube due to the ${ }^{113} \mathrm{Cd}(\mathrm{n}, \gamma){ }^{114} \mathrm{Cd}$ capture reaction. The alpha and beta responses were measured with an ${ }^{241} \mathrm{Am}$ source and a ${ }^{36} \mathrm{Cl}$ source, respectively, with the sources placed directly against the sample. The peaks were recorded for the alpha responses and the end points were recorded for the beta responses. Gamma-ray responses were obtained using a $1 \mu \mathrm{Ci}{ }^{60} \mathrm{Co}$ source. The light pulses from the samples were converted into electrical pulses using a Philips 2202B PMT mounted on a Canberra 2007P base powered by an ORTEC 556 high voltage power supply set at $1200 \mathrm{~V}$. The signals from the base were amplified using an ORTEC 572A amplifier set at $50 \mathrm{G}$ with a $2 \mu$ s shaping time. The amplified signal was digitalized using an ORTEC $926 \mathrm{MCB}$ with an 8192 channel ADC. The digitalized output was then saved using the MAESTRO-32 software from ORTEC.

\section{Results and Discussion}

3.1. Fluorescence Characterizations. A typical normalized fluorescence spectrum of a sample containing PS, ${ }^{6} \mathrm{LiF}$, and PPO/POPOP is shown in Figure 4. The wavelength of maximum excitation $\left(\lambda_{\mathrm{ex}}=274 \mathrm{~nm}\right)$ is characteristic of PS 


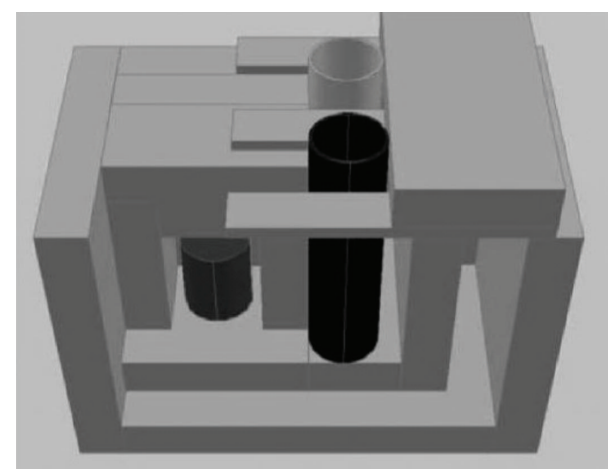

Figure 3: Representation of the neutron irradiator. The small cylinder represents a 0.59 microgram ${ }^{252} \mathrm{Cf}$ source surrounded by $0.5 \mathrm{~cm}$ of stainless steel and $1.25 \mathrm{~cm}$ of lead. The neutrons are moderated by $5 \mathrm{~cm}$ high density polyethylene (HDPE) to moderate the neutrons. The black tube represents an acrylic tube $1.6 \mathrm{~mm}$ thick wrapped with a $1.6 \mathrm{~mm}$ thick sheet of cadmium. The lighter tube represents an acrylic tube $1.6 \mathrm{~mm}$ thick wrapped with $1.6 \mathrm{~mm}$ lead. The entire irradiator is housed in $5 \mathrm{~cm}$ thick HDPE. A side of the irradiator has been removed in the image for clarity.

excitation. The wavelength of maximum emission $\left(\lambda_{\mathrm{em}}=\right.$ $421 \mathrm{~nm}$ ) is characteristic of POPOP emission. A photophysical mechanism for the possible routes of excitation enegy migration is shown in the following.

(1) Excitation of Matrix

$$
\mathrm{PS}+\lambda_{1} \longrightarrow \mathrm{PS}^{*}
$$

(2) Matrix Relaxation

$$
\begin{aligned}
& \mathrm{PS}^{*} \longrightarrow \mathrm{PS} \\
& \mathrm{PS}^{*} \longrightarrow \mathrm{PS}+\lambda_{2} \quad\left(\phi_{f}=0.16\right) \\
& \mathrm{PS}^{*}+\mathrm{PPO} \longrightarrow \mathrm{PS}+\mathrm{PPO}^{*}
\end{aligned}
$$

(3) PPO Excitation

$$
\begin{aligned}
& \mathrm{PS}^{*}+\mathrm{PPO} \longrightarrow \mathrm{PS}+\mathrm{PPO}^{*} \\
& \mathrm{PPO}+\lambda_{2} \longrightarrow \mathrm{PPO}^{*}
\end{aligned}
$$

(4) PPO Relaxation

$$
\mathrm{PPO}^{*} \longrightarrow \mathrm{PPO}+\lambda_{3} \quad\left(\phi_{f}=1.00\right)
$$

(5) POPOP Excitation

$$
\lambda_{3}+\text { POPOP } \longrightarrow \text { POPOP }^{*}
$$

(6) POPOP Relaxation

$$
\begin{aligned}
& \text { POPOP* }^{*} \longrightarrow \text { POPOP } \\
& \text { POPOP }^{*} \longrightarrow \text { POPOP }+\lambda_{4} \quad\left(\phi_{f}=0.93\right),
\end{aligned}
$$

where $\phi_{f}$ is quantum efficiency of fluorescence [10], $\lambda_{i}$ represents a photon with a wavelength characteristic of the process, and $*$ indicates an electronically excited state. First PS is excited at $274 \mathrm{~nm}$. Ideally, the excitation energy is

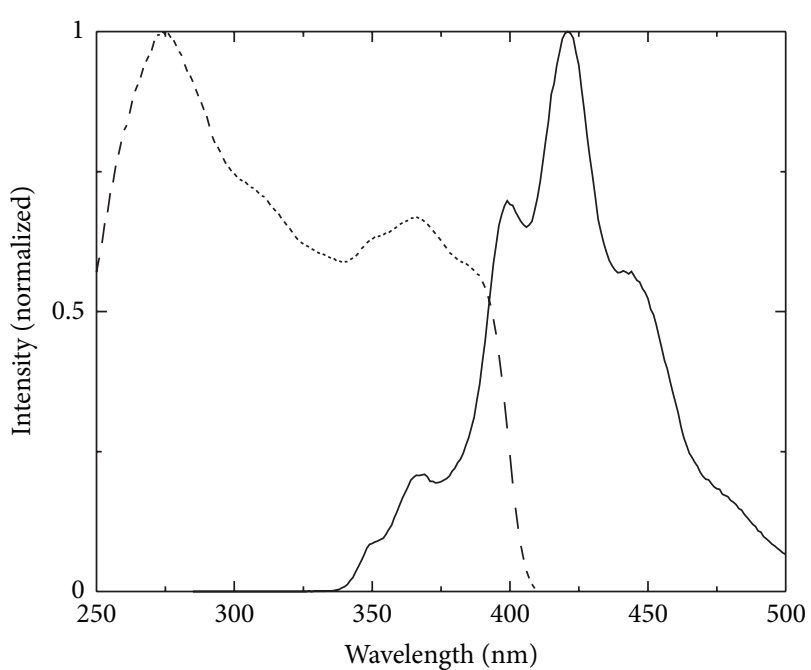

Figure 4: Excitation (--) and emission (-) spectra of a typical sample. Spectra are normalized to maximum peak intensities.

transferred nonradiatively from PS by PPO, which then emits a photon with $100 \%$ quantum efficiency. This photon is then absorbed by POPOP and reemitted at $421 \mathrm{~nm}$ with a quantum efficiency of $93 \%$.

The efficiency of excitation of PS is dictated by the extinction coefficient at the wavelength of maximum excitation which is an intrinsic property of the material. After the PS is excited, there are three competing processes that determine the fate of the excitation energy: the excited monomer unit can (1) be quenched internally, (2) emit a photon at $\lambda_{2}$ (excimer emission), or (3) transfer the energy to PPO. Transfer of excitation energy between PS and PPO occurs by both radiative and nonradiative mechanisms; the fraction of nonradiative transfer increases with increasing concentration of PPO [11].

Before incorporation of ${ }^{6} \mathrm{LiF}$ in the matrix, it was first necessary to optimize the concentration of PPO/POPOP to maximize the light yield. Fluorescence spectra of PS containing varying concentrations of PPO/POPOP (Figure 5) were analyzed to determine the concentrations most suitable for use in these samples. To maximize the light yield, it was first necessary to ensure that the maximum amount of excitation energy is collected from the PS matrix. By measuring the excitation intensity that results in emission from PS $(314 \mathrm{~nm})$ as a function of PPO/POPOP concentration, the concentration at which no PS emission is observed can be determined. This should be the concentration at which transfer to PPO is optimum and no light is lost as emission from PS. The data in Figure 5 show that the emission from PS decreases rapidly with the addition of low concentrations of PPO/POPOP. At $1.5 \%$ the emission from PS is reduced by approximately $96 \%$.

The concentration at which sufficient fluor molecules are present to harvest the excitation energy from PS such that no emission from PS is observed should be optimized for use as a scintillation composition. However, considering that quantum efficiency of a pure PS film is about 0.16 , it is possible 


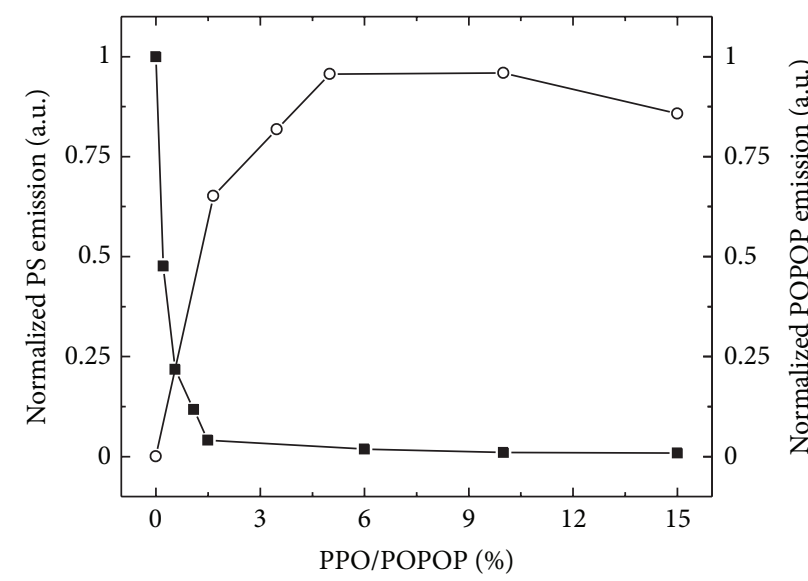

Figure 5: PS emission ( $\mathbf{a}$ ) and POPOP emission (o) for $150 \mu \mathrm{m}$ samples as a function of $w \mathrm{t} \% \mathrm{PPO} / \mathrm{POPOP}$. PS emission was obtained by measuring the excitation spectrum that yielded emission at $314 \mathrm{~nm}$, then integrating the response over the region $250 \mathrm{~nm}-300 \mathrm{~nm}$. POPOP emission was obtained by measuring the emission spectrum resulting from excitation at $274 \mathrm{~nm}$, then integrating over the region $390 \mathrm{~nm}-500 \mathrm{~nm}$.

that more excitation energy can be collected by further increasing the fluor concentration. To test this hypothesis, PS was excited at $274 \mathrm{~nm}$ and the emission from POPOP was measured as a function of PPO/POPOP concentration. It can be seen that although $96 \%$ of the PS emission is quenched at $1.5 \%$, only $75 \%$ of the maximum emission intensity from POPOP is achieved at this concentration. Further addition of $\mathrm{PPO} / \mathrm{POPOP}$ results in an increase in emission intensity up to approximately $5 \%$. The emission levels off until $10 \%$, then decreases thereafter due to selfabsorption. This demonstrates that the optimum concentration of $\mathrm{PPO} / \mathrm{POPOP}$ in this material is in the range $5-10 \%$. It is interesting to note that the concentration at which nearly all PS emission is quenched, addition of more fluor molecules significantly increases the amount of excitation energy that can be collected from PS. This effectively increases the overall quantum efficiency of the system. It is thus determined that even though the overall quantum efficiency of the pure matrix is low, the quantum efficiency of the overall emission can be improved by utilization of a fluor to which PS efficiently transfers excitations before vibrational relaxation occurs.

It is generally agreed upon that effective scintillators must be transparent to their own scintillation light. To determine the effect of reduced optical transmission on fluorescence, emission spectra shown in Figure 6 were measured for samples containing 10\% PPO/POPOP with varying concentrations of ${ }^{6} \mathrm{LiF}$. Comparison of the relative emission intensities demonstrates that increasing the amount of ${ }^{6} \mathrm{LiF}$ in the sample increases the emission intensity. This can be rationalized by considering that the $\mathrm{PS} /{ }^{6} \mathrm{LiF}$ composite is a mechanical mixture with $3.2 \mu \mathrm{m}$ cubic lithium fluoride particles trapped inside the PS matrix. Because the refractive indices of lithium fluoride and polystyrene are different and the particles are sufficiently larger than the wavelength of incident light, each particle acts as a scattering site for the

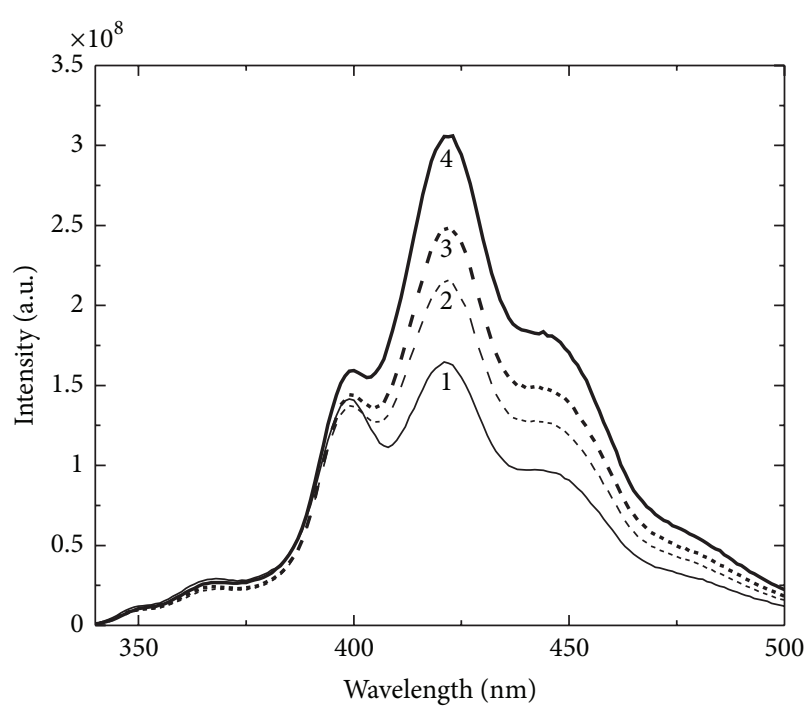

FIGURE 6: Emission spectra of $150 \mu \mathrm{m}$ thick samples containing 10\% $\mathrm{PPO} / \mathrm{POPOP}$ at various loadings of ${ }^{6} \mathrm{LiF}$. Spectra were obtained by excitation at $274 \mathrm{~nm}$. Spectra are numbered according to the following percentages of ${ }^{6} \mathrm{LiF}$ : (1) $0 \%$, (2) $10 \%$, (3) $15 \%$, and (4) $20 \%$.

incident excitation photons. A greater number of scattering sites in the matrix increases the overall path length of any given photon in the film. The Beer-Lambert law indicates that absorption of a photon by a material is dependent on both concentration and path length of the absorbing medium. It was shown in Figure 4 that increasing the concentration of $\mathrm{PPO} / \mathrm{POPOP}$ above $10 \%$ results in a decrease in the observed emission, so the observed phenomenon in Figure 5 cannot be due to a higher probability of scattered photons emitted from PPO being captured by POPOP. This also cannot be attributed to the energy transfer between PS and PPO because this mechanism occurs primarily without emission of a photon. Thus, most probable explanation for this phenomenon is that the greater number of scattering sites increases the average path length of the photons in the excitation beam through the material, resulting in a greater probability that an incident photon will be absorbed by the PS. Because more photons are absorbed in PS matrices with higher loadings of ${ }^{6} \mathrm{LiF}$, more excitations are available to be harvested by the antenna fluors.

3.2. Scintillation Measurements. The films were characterized by responses to alpha radiation from ${ }^{241} \mathrm{Am}$. Alpha responses of films with different percentages of fluor $(0.1-15 \%)$ were measured for $50 \mu \mathrm{m}$ and $150 \mu \mathrm{m}$ samples. Peak positions indicated that the optimum concentration of fluor was $5 \%$, which is in agreement with the fluorescence data. This weight percentage was used throughout the rest of the experimentation. Alpha responses of samples containing $10 \%{ }^{6} \mathrm{LiF}$ and $5 \%$ $\mathrm{PPO} / \mathrm{POPOP}$ with thicknesses $15 \mu \mathrm{m}-150 \mu \mathrm{m}$ are shown in Figure 7. It is observed that thinner samples have a decreased resolution and therefore a decreased average light output as characterized by the broad peaks whereas the thicker samples show a sharper peak and therefore a greater average light output. This is attributed to the fact that the range of the 


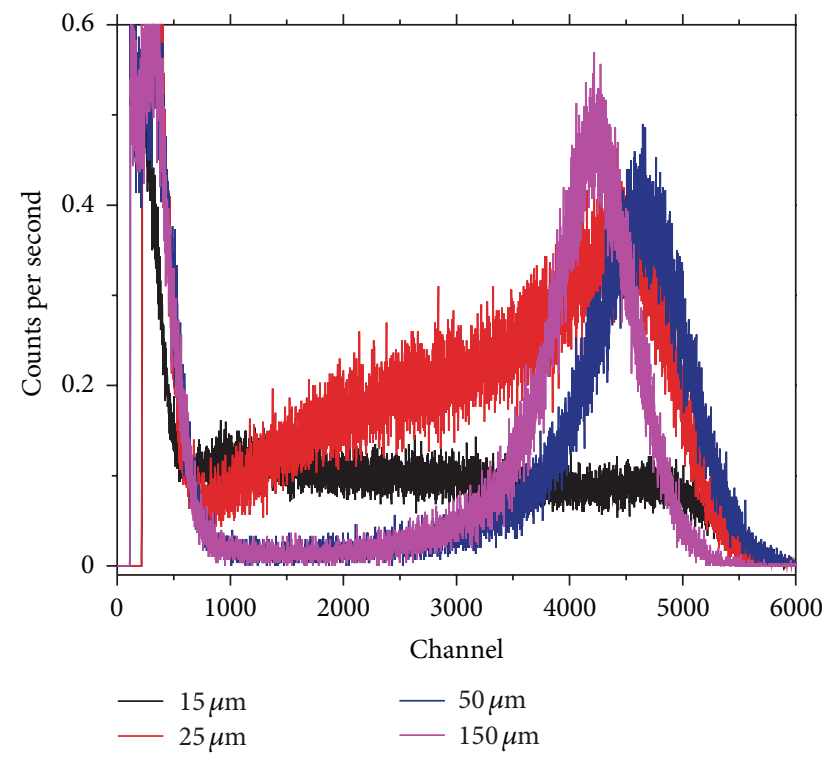

Figure 7: Alpha spectra for samples containing $10 \%{ }^{6} \mathrm{LiF}$ and $5 \%$ $\mathrm{PPO} / \mathrm{POPOP}$ for thicknesses over the range 15-150 $\mu \mathrm{m}$.

TABLE 1: Ranges of charged particles.

\begin{tabular}{lccc}
\hline Material & $\alpha^{2+}(5.48 \mathrm{MeV})$ & $\alpha^{2+}(2.05 \mathrm{MeV})$ & $t^{+}(2.73 \mathrm{MeV})$ \\
\hline PS & $39.1 \mu \mathrm{m}$ & $10.1 \mu \mathrm{m}$ & $61.8 \mu \mathrm{m}$ \\
${ }^{6} \mathrm{LiF}^{\mathrm{a}}$ & $20.8 \mu \mathrm{m}$ & $5.81 \mu \mathrm{m}$ & $33.1 \mu \mathrm{m}$ \\
\hline
\end{tabular}

Ranges of charged particles were calculated using SRIM-2011 [12]. Reported values are averages from simulating 500 ion interactions.

${ }^{\text {a }}$ Density and molar mass of ${ }^{6} \mathrm{LiF}$ were adjusted for $97.6 \%{ }^{6} \mathrm{Li}$ enrichment.

incident $5.48 \mathrm{MeV}$ alpha is about $39.1 \mu \mathrm{m}$ in PS, as shown in Table 1, which exceeds the thicknesses of the 15 and $25 \mu \mathrm{m}$ thick films. This indicates that the incident alphas are not completely stopped in the 15 and $25 \mu \mathrm{m}$ films, whereas they are completely stopped in the 50 and $150 \mu \mathrm{m}$ films. Thus, it is expected that the resolution should be poor in the 15 and $25 \mu \mathrm{m}$ films and improved in the 50 and $150 \mu \mathrm{m}$ films. It can also be seen that the light output is reduced in the $150 \mu \mathrm{m}$ film relative to the $50 \mu \mathrm{m}$ film. This is attributed to increased self-absorption and increasing scattering probability of the scintillation light in the $150 \mu \mathrm{m}$ sample. The active volume is only about $39 \mu \mathrm{m}$ deep as evidenced by the range of the alpha; thus, the scintillation light must travel a greater distance in the $150 \mu \mathrm{m}$ film to reach the PMT as compared to the $50 \mu \mathrm{m}$ film, increasing the probability that self-absorption or scattering will occur.

In order to estimate the repeatability in fabrication and measurement of these samples, four compositions were fabricated multiple times and the neutron count rates in counts per second (CPS) were recorded. The compositions were $25 \mu \mathrm{m}$ and $50 \mu \mathrm{m}$ samples each containing $20 \%$ and $30 \%{ }^{6} \mathrm{LiF}$ with 5\% PPO/POPOP. These results (Table 2) are included to indicate the level of repeatability for net neutron count rates for identically prepared and counted samples. Differences in net neutron count rates are attributed to potential changes
TABLE 2: Repeatability of neutron count rates.

\begin{tabular}{lcc}
\hline Thickness & wt $\%{ }^{6} \mathrm{LiF}$ & Neutron Count Rate (CPS) \\
\hline \multirow{2}{*}{$25 \mu \mathrm{m}$} & 20 & $20.9 \pm 3.6$ \\
& 30 & $46.9 \pm 1.5$ \\
\hline \multirow{2}{*}{$50 \mu \mathrm{m}$} & 20 & $52.2 \pm 2.6$ \\
& 30 & $91.4 \pm 2.7$ \\
\hline
\end{tabular}

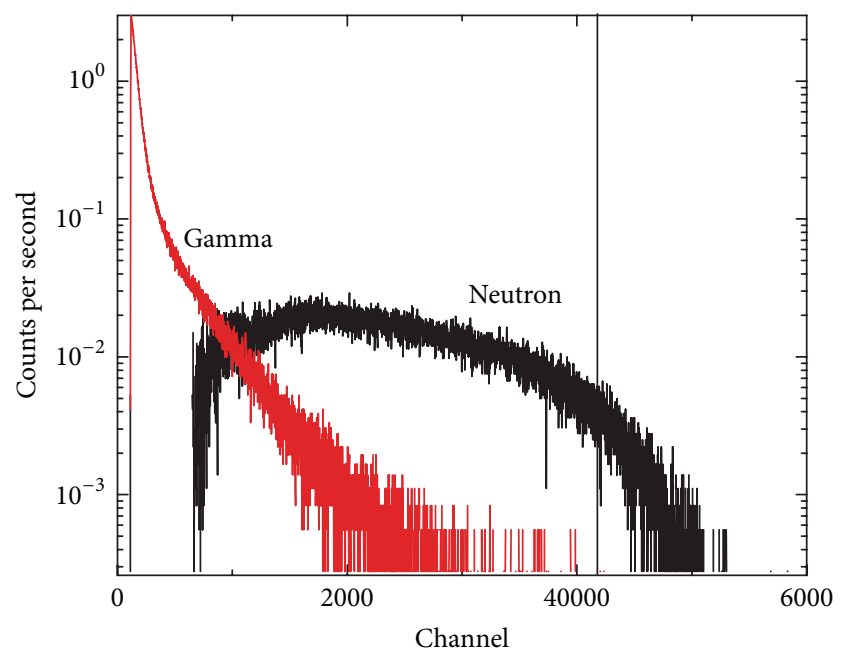

FIGURE 8: Neutron (black line) and gamma-ray (red line) responses for a $50 \mu \mathrm{m}$ sample containing $20 \%{ }^{6} \mathrm{LiF}$ and $5 \% \mathrm{PPO} / \mathrm{POPOP}$. The vertical line indicates the lower-level discriminator setting at which the neutron intrinsic efficiency is $1.2 \times 10^{-3}$. At this point, the gamma-ray intrinsic efficiency is $5.24 \times 10^{-6}$, indicating that approximately 1 in every 190,000 gamma-rays passing through the detector surface is misclassified as a neutron.

in dispersion of ${ }^{6} \mathrm{LiF}$, inherent error in measuring submilligram quantities during sample fabrication, and small changes in the geometry among multiple measurements. The error values indicate that the measurement and fabrication protocols are repeatable.

Neutron and gamma-ray responses for a $50 \mu \mathrm{m}$ thick sample containing $20 \%{ }^{6} \mathrm{LiF}$ and $5 \% \mathrm{PPO} / \mathrm{POPOP}$ are shown in Figure 8. The separation of the neutron and gamma-ray spectra indicate that neutron/gamma-ray discrimination can be achieved in these samples using pulse height discrimination techniques. The absence of a clear peak can be attributed to the ranges of the fission products, shown in Table 1, relative to the film thickness. The average range of the triton in PS is approximately $60 \mu \mathrm{m}$ which is greater than the thickness of the film. Assuming that the neutron capture probability does not vary over the entire thickness of the film [7], it is likely that a significant fraction of the energy of the triton is not deposited in the film. This decreases the overall resolution of the composite material; however, thicker samples result in more overlap of the neutron and gamma-ray spectra, thereby eliminating the possibility of discrimination.

Pulse height discrimination between neutron and gamma-ray events is facilitated when the neutron count rates at higher channel numbers are increased, indicative of higher efficiency detection of charged particles. Samples 
containing higher loadings of ${ }^{6} \mathrm{LiF}$ have higher total count rates. However, at higher channels the samples with higher loadings become less efficient due to scattering. This can be rationalized by considering two factors present in the different samples. First, higher concentrations of ${ }^{6} \mathrm{LiF}$ result in a decrease in optical clarity of the samples. This increases the probability that the scintillation light will be scattered before reaching the photomultiplier tube, resulting in a lower observed efficiency. Additionally, it must be considered that lithium fluoride does not scintillate and energy deposited in the lithium fluoride particles is not efficiently transferred to the scintillating volume. Increasing the fraction of nonscintillating particles in the matrix thus decreases the overall kinetic energy available to be transduced into scintillation light.

Values for discriminator (PHD) settings were obtained by integrating the neutron and gamma-ray spectra, dividing each point by the total fluence (1050 neutrons/s and $1850 \mathrm{\gamma} / \mathrm{s}$ ), then finding the channel at which the intrinsic neutron efficiency is $1.2 \times 10^{-3}$ and the gamma-ray efficiency is $1 \times$ $10^{-6}$, as shown in Figure 9. The data in Figure 9 indicate the pulse height discriminator level required to achieve intrinsic neutron efficiencies of $1.2 \times 10^{-3}$ and intrinsic gamma-ray detection efficiencies of $1 \times 10^{-6}$ for samples containing $10 \%{ }^{6} \mathrm{LiF}$ and $5 \% \mathrm{PPO} / \mathrm{POPOP}$ over the thickness range $15-2600 \mu \mathrm{m}$. To maintain an intrinsic neutron detection efficiency of $1.2 \times 10^{-3}$, the lower-level discriminator setting must be placed at channel 4234 . At this setting, the intrinsic gamma-ray detection efficiency is $5.24 \times 10^{-6}$. This indicates that if a lower-level discriminator setting is placed at this point, the intrinsic neutron detection efficiency will be 1.2 $\times 10^{-3}$ and approximately 1 out of every 190,000 gammarays passing through the detector will be misclassified as a neutron.

It is evident from the plot shown in Figure 10 that the best neutron/gamma-ray discrimination can be achieved with samples in the thickness range $25 \mu \mathrm{m}-150 \mu \mathrm{m}$. Above $150 \mu \mathrm{m}$, the material becomes more sensitive to gamma-rays and below $15 \mu \mathrm{m}$ the range of the charged particles released from ${ }^{6} \mathrm{Li}$ fission greatly exceeds the thickness of the film such that a sharp reduction in the pulse height is observed.

Sample geometry required to achieve neutron/gammaray discrimination is at the expense of neutron detection efficiency. Shown in Figure 11 are the intrinsic efficiencies for the samples reported in Figure 10. It is demonstrated that even though thinner samples demonstrate better neutron/gammaray discrimination capabilities, it is achieved at the expense of intrinsic neutron detection efficiencies. Thicker samples can be fabricated to achieve detection efficiencies of up to $70 \%$ but this comes at the expense of the material to facilitate neutron/gamma-ray discrimination.

\section{Conclusions}

This work has demonstrated that effective composite thermal neutron scintillation detectors can be fabricated using PS, ${ }^{6} \mathrm{LiF}$, and $\mathrm{PPO} / \mathrm{POPOP}$ and cast as films by solution

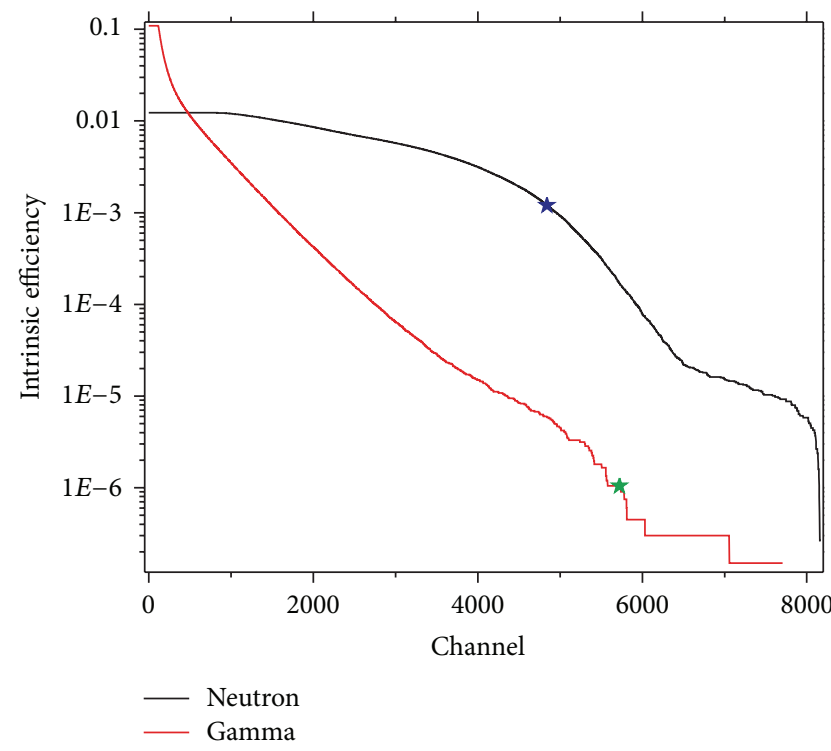

FIGURE 9: Intrinsic efficiency plotted against channel number for a $50 \mu \mathrm{m}$ film containing $10 \%{ }^{6} \mathrm{LiF}$ and $5 \%$ PPO/POPOP. Stars indicate the channels at which intrinsic efficiencies reach $1.2 \times 10^{-3}$ for neutrons and $10^{-6}$ for gamma-rays.

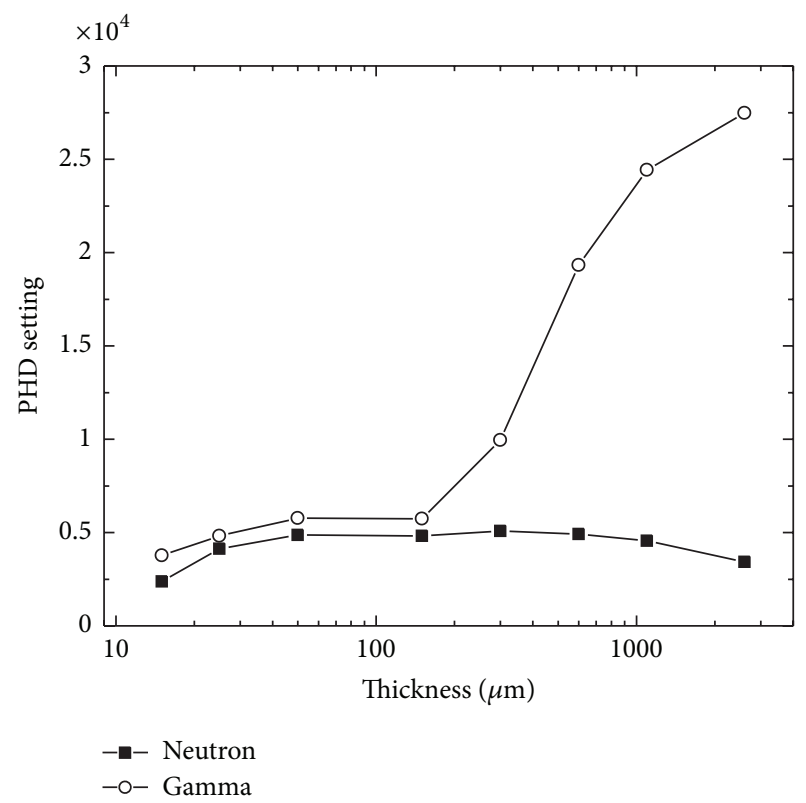

FIgURE 10: PHD levels required to achieve intrinsic neutron efficiencies of $1.2 \times 10^{-3}$ and intrinsic gamma-ray-neutron detection efficiencies of $1 \times 10^{-6}$ as a function of thickness. The optimal discrimination capabilities can be achieved with materials in the range $25 \mu \mathrm{m}-150 \mu \mathrm{m}$.

casting methods. Compositions of the films were varied to optimize responses. Fluorescence intensity for neat films was found to be optimum at 5\% PPO/POPOP and was improved by adding ${ }^{6} \mathrm{LiF}$ to act as scattering sites for the incident excitation beam. The gamma-ray response was unaffected by changing concentration of ${ }^{6} \mathrm{LiF}$ in the matrix. The 


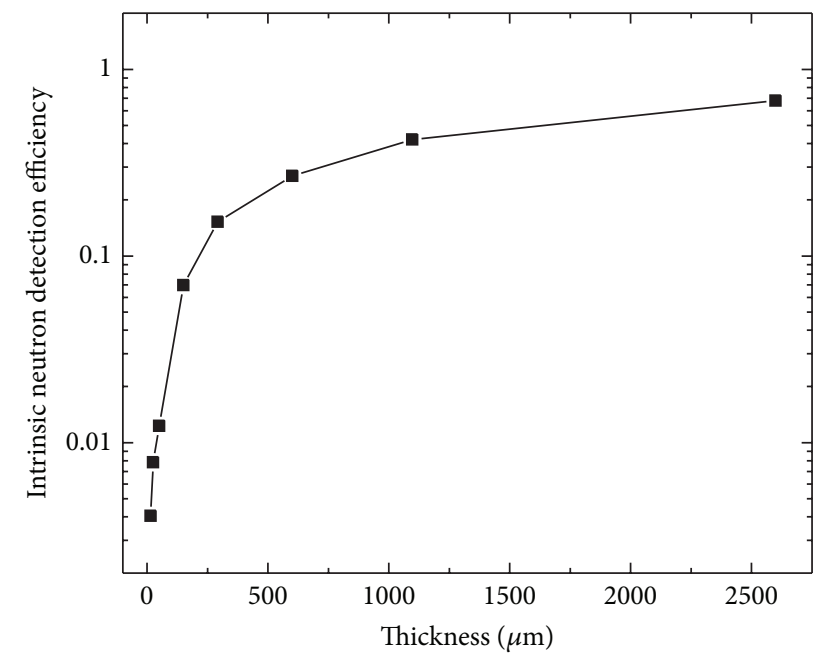

FIGURE 11: Intrinsic neutron detection efficiency as a function of thickness for samples containing $10 \%{ }^{6} \mathrm{LiF}$ and 5\% PPO/POPOP.

best neutron/gamma-ray discrimination capabilities were obtained in the film thickness range $25-150 \mu \mathrm{m}$, though the discrimination capabilities are inferior compared to ${ }^{3} \mathrm{He}$ based systems. It is shown that for films containing $10 \%{ }^{6} \mathrm{LiF}$, intrinsic neutron detection efficiencies approach $70 \%$ but this comes at the expense of reduced neutron/gamma-ray discrimination capabilities.

\section{Conflict of Interests}

None of the authors has any direct financial relation with Teflon or any other commercial identity mentioned in this paper.

\section{Acknowledgments}

This work was supported by the Domestic Nuclear Detection Office (DNDO) through award 003387891. Any opinions, findings, and conclusions or recommendations expressed in this material are those of the authors and do not necessarily reflect the views of DNDO. The authors gratefully acknowledge the Center for Mass Spectrometry at the University of Tennessee for assistance in determination of ${ }^{6} \mathrm{Li} /{ }^{7} \mathrm{Li}$ ratios. Dr. Dayakar Penumadu would like to acknowledge Dr. Steven Wallace from Neutron Sciences for providing ${ }^{6} \mathrm{Li}$ through prior collaborations with the University of Tennessee.

\section{References}

[1] R. T. Kouzes, The ${ }^{3}$ He Supply Problem. PNNL-18388, Pacific Northwest National Laboratory, Richland, Wash, USA, 2009.

[2] A. Quaranta, S. M. Carturan, T. Marchi et al., "Optical and scintillation properties of polydimethyl-diphenylsiloxane based organic scintillators," IEEE Transactions on Nuclear Science, vol. 57, no. 2, pp. 891-900, 2010.

[3] R. K. Swank and W. L. Buck, "The scintillation process in plastic solid solutions," Physical Review, vol. 91, no. 4, pp. 927-933, 1953.
[4] W. S. Koski, "Scintillations in some phosphor-plastic systems," Physical Review, vol. 82, no. 2, pp. 230-232, 1950.

[5] J. B. Czirr, G. M. MacGillivray, R. R. MacGillivray, and P. J. Seddon, "Performance and characteristics of a new scintillator," Nuclear Instruments and Methods in Physics Research A, vol. 424, no. 1, pp. 15-19, 1999.

[6] G. F. Knoll, Radiation Detection and Measurement, John Wiley \& Sons, New York, NY, USA, 4th edition, 2010.

[7] I. Sen, D. Penumadu, M. Williamson, L. F. Miller, A. D. Green, and A. N. Mabe, "Thermal neutron scintillator detectors based on poly (2-vinylnaphthalene) composite films," IEEE Transactions on Nuclear Science, vol. 58, no. 3, pp. 1386-1393, 2011.

[8] I. Sen, M. Urffer, D. Penumadu, S. A. Young, L. F. Miller, and A. N. Mabe, "Polyester composite thermal neutron scintillation films," IEEE Transactions on Nuclear Science, vol. 59, no. 4, pp. 1781-1786, 2012.

[9] R. T. Kouzes, J. R. Ely, A. T. Lintereur, and D. L. Stephens, PNNL18903: Neutron Detector Gamma Insensitivity Criteria, Pacific Northwest National Laboratory, Richland, Wash, USA, 2009.

[10] I. B. Berlman, Handbook of Fluorescence Spectra of Aromatic Molecules, Academic Press, New York, NY, USA, 2nd edition, 1971.

[11] M. Leibowitz and A. Weinreb, "Effects of fluorescence and energy transfer in polystyrene under excitation in the vacuum ultraviolet," The Journal of Chemical Physics, vol. 46, no. 12, pp. 4652-4659, 1967.

[12] J. F. Ziegler, M. D. Ziegler, and J. P. Biersack, "SRIM-the stopping and range of ions in matter (2010)," Nuclear Instruments and Methods in Physics Research B, vol. 268, no. 11-12, pp. 1818$1823,2010$. 

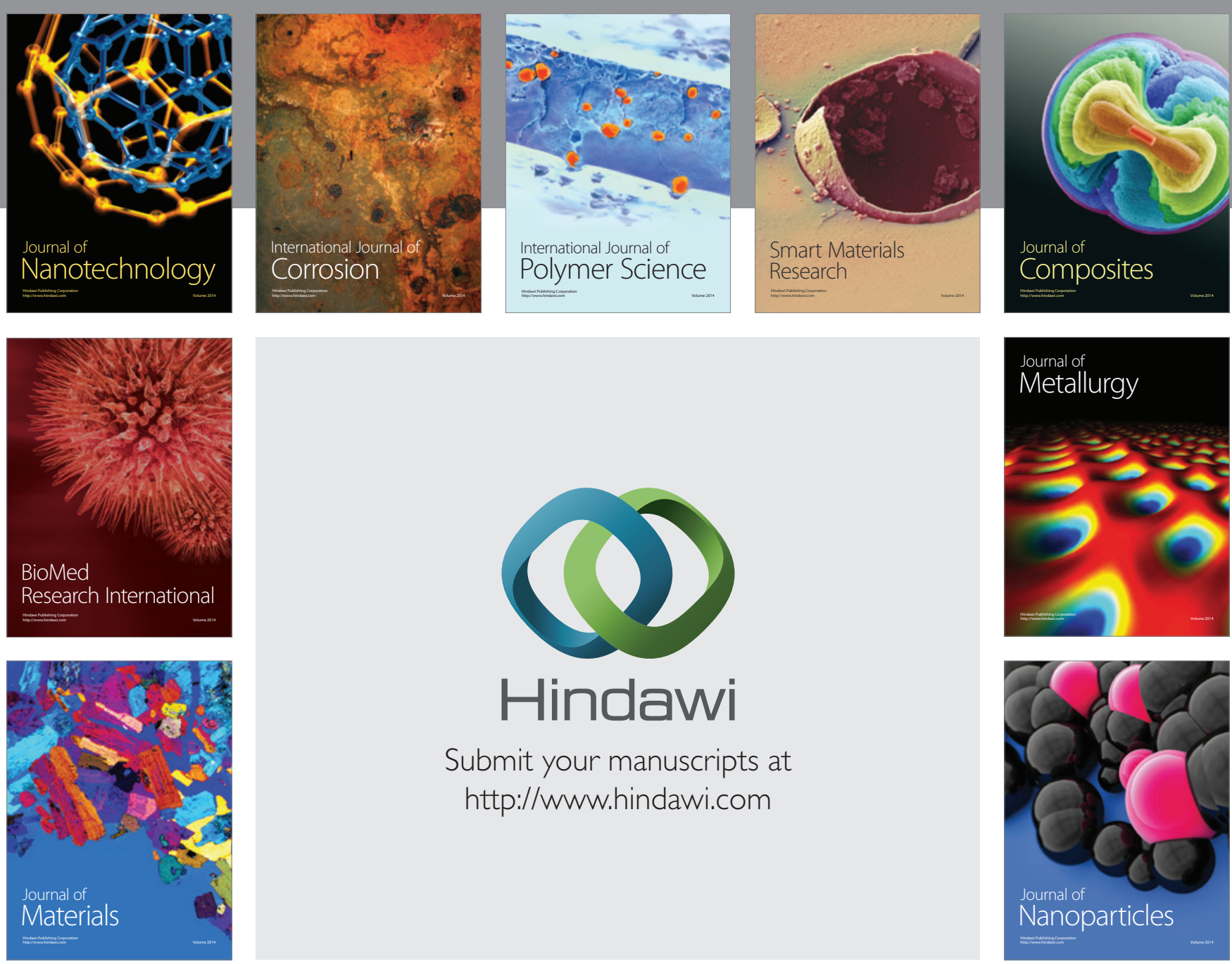

Submit your manuscripts at http://www.hindawi.com
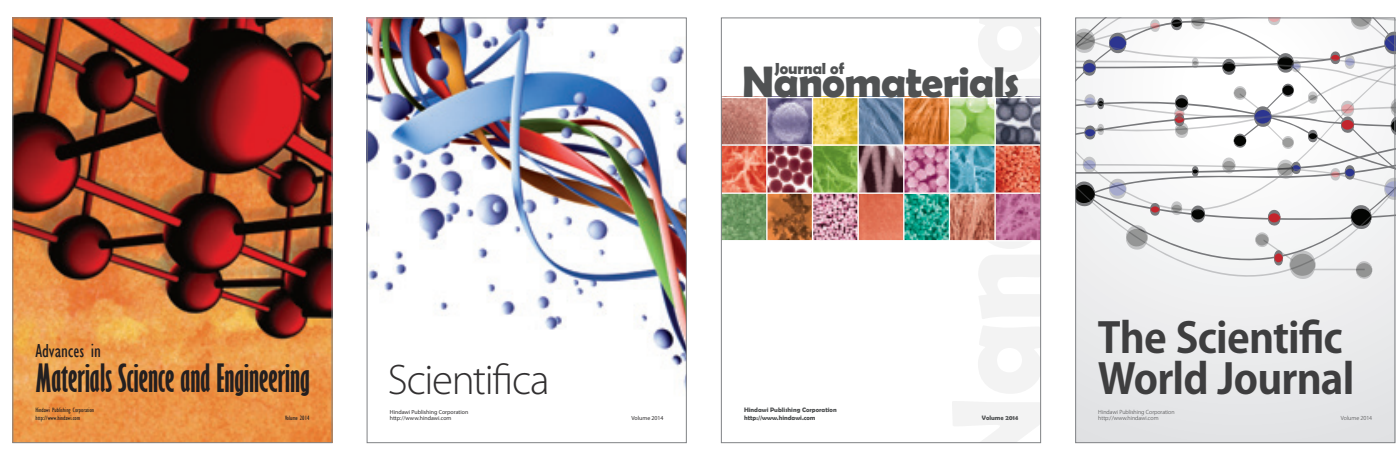

\section{The Scientific World Journal}
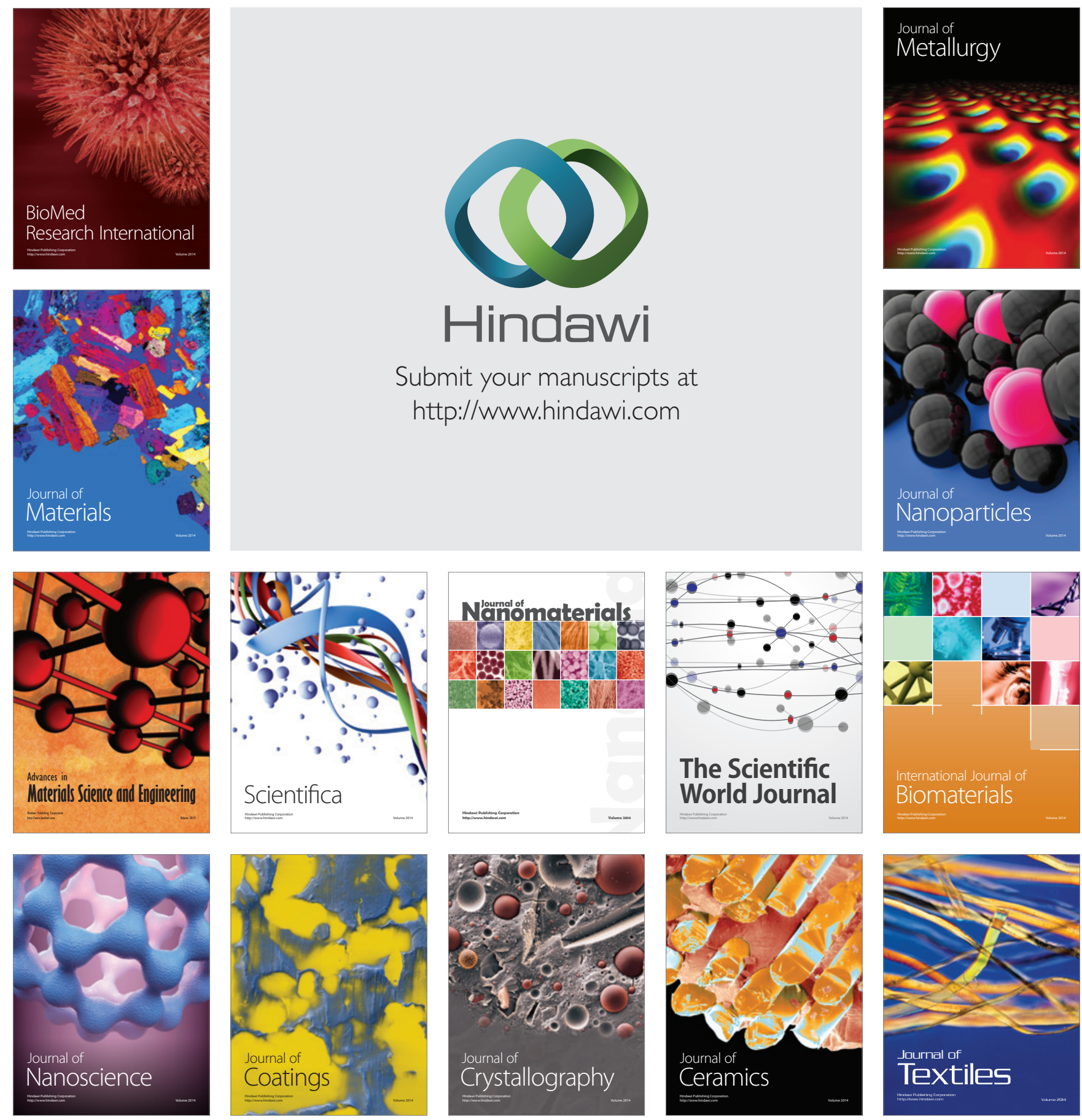